\title{
Electrochemical Estimation of the Corrosion Rate of Magnesium/Aluminium Alloys
}

\author{
A. Pardo, ${ }^{1}$ S. Feliu Jr., ${ }^{2}$ M. C. Merino, ${ }^{1}$ R. Arrabal, ${ }^{1}$ and E. Matykina ${ }^{3}$ \\ ${ }^{1}$ Departamento de Ciencia de Materiales, Facultad de Química, Universidad Complutense, 28040 Madrid, Spain \\ ${ }^{2}$ Centro Nacional de Investigaciones Metalúrgicas, CSIC, Avda. Gregorio del Amo 8, 28040 Madrid, Spain \\ ${ }^{3}$ Corrosion and Protection Centre, School of Materials, The University of Manchester, P.O. Box 88, Sackville Street, \\ Manchester M60 1QD, UK \\ Correspondence should be addressed to A. Pardo, anpardo@quim.ucm.es
}

Received 19 June 2009; Accepted 5 October 2009

Academic Editor: Raman Singh

Copyright (๑) 2010 A. Pardo et al. This is an open access article distributed under the Creative Commons Attribution License, which permits unrestricted use, distribution, and reproduction in any medium, provided the original work is properly cited.

The corrosion rate of AZ31, AZ80, and AZ91D magnesium/aluminium alloys immersed in 3.5 wt.\% NaCl was determined comparing gravimetric and electrochemical measurements. The findings revealed that, for all investigated materials, a fraction of the metallic surface exposed to the corrosive medium did not reveal a normal electrochemical response to the applied signal. This may be associated with phenomena such as partial disintegration of specimens into fine metallic particles, electrochemical formation of $\mathrm{Mg}^{+}$ions, and/or anomalous chemical attack occurring simultaneously with the normal electrochemical corrosion attack. The abnormal electrochemical behaviour was more evident for lower amounts of aluminium in the bulk composition of the investigated materials. Thus, the electrochemical estimates of pure Mg and the AZ31 alloy were not reliable and tended to underestimate corrosion losses.

\section{Introduction}

Corrosion susceptibility restricts the use of magnesium alloys in many engineering applications, especially in chloridecontaining media. The corrosion behaviour of magnesium alloys depends on the medium which they are exposed to, but it is also strongly influenced by the bulk composition and the microstructure of the alloy. Thus, for an adequate use of magnesium materials, corrosion studies are fundamental, which normally involve electrochemical methods. One of the main advantages of electrochemical measurements is the continuous monitoring of the corrosion process during the exposure time. The standard behaviour of metals immersed in corrosive media implies that the corrosion rates obtained from gravimetric and electrochemical tests coincide, which is indicative of a corrosive process due to electrochemical dissolution only. Makar and Kruger [1] found a satisfying consistency between both types of tests in the case of uniformly attacked magnesium alloys immersed in a sodium borate solution, but not for pure magnesium, which disclosed nonuniform attack. Similar discrepancies were revealed between electrochemical and gravimetric results for other magnesium alloys immersed in other corrosive media, which were associated with various causes such as material disintegration, participation of monovalent $\mathrm{Mg}^{+}$ ions, anomalous chemical dissolution, and limitations in the application of the Stern-Geary equation [1-5].

Occasionally, electrochemical corrosion of magnesium in aqueous solutions is accompanied by breaking up of the metal into fine particles, which significantly contributes to corrosion damage of magnesium [1-5]. This phenomenon has been known for a long time and is due to particular ways of attack penetration, that is, the presence of a less corrosion resistant phase with enough geometric continuity that triggers undermining of other phases. In the case of magnesium alloys, grain boundaries are normally cathodic compared with the body of the grains. Thus, the corrosion attack is concentrated on the anodic area adjoining the boundary until, eventually, metallic particles surrounded by the attack fall out of the matrix [6].

The existence of monovalent magnesium ion $\left(\mathrm{Mg}^{+}\right)$as an intermediate specie has also been proposed $[3,7-13]$. 
TABLE 1: Nominal composition of the tested materials.

\begin{tabular}{|c|c|c|c|c|c|c|c|c|c|c|}
\hline \multirow{2}{*}{ Material } & \multicolumn{10}{|c|}{ Chemical composition (wt.\%) } \\
\hline & $\mathrm{Al}$ & $\mathrm{Zn}$ & $\mathrm{Mn}$ & $\mathrm{Si}$ & $\mathrm{Cu}$ & $\mathrm{Fe}$ & $\mathrm{Ni}$ & $\mathrm{Ca}$ & $\mathrm{Zr}$ & Others \\
\hline $\operatorname{Mg}(99 \%)$ & 0.006 & 0.014 & 0.03 & 0.019 & 0.001 & 0.004 & $<0.001$ & & & \\
\hline AZ31 & 3.1 & 0.73 & 0.25 & 0.02 & $<0.001$ & 0.005 & $<0.001$ & $<0.01$ & $<0.001$ & $<0.30$ \\
\hline AZ80 & 8.2 & 0.46 & 0.13 & 0.01 & $<0.001$ & 0.004 & & & & $<0.30$ \\
\hline AZ91D & 8.8 & 0.68 & 0.30 & 0.01 & $<0.001$ & 0.004 & $<0.008$ & & & $<0.30$ \\
\hline
\end{tabular}

In particular, the presence of this ion has been used to explain the "negative difference effect", which is related to the accelerated hydrogen evolution rate with increasing anodic overvoltage. This is a rather common phenomenon during anodic dissolution of magnesium and its alloys $[5,10]$. It is assumed that, to some degree, monovalent magnesium ions are produced electrochemically according to the reaction

$$
\mathrm{Mg}=\mathrm{Mg}^{+}+\mathrm{e}^{-} .
$$

$\mathrm{Mg}^{+}$ions chemically react with water to produce divalent magnesium ions $\left(\mathrm{Mg}^{2+}\right)$ and hydrogen:

$$
2 \mathrm{Mg}^{+}+\mathrm{H}_{2} \mathrm{O}=2 \mathrm{Mg}^{2+}+2 \mathrm{OH}^{-}+\mathrm{H}_{2} .
$$

The latter reaction does not imply transfer of electrons across the metal/solution interface. Thus, it is not reflected in the electrochemical estimates of corrosion, which will be underrated.

Another possible explanation for electrochemical corrosion determinations several and even many times lower than the corrosion rates directly determined by gravimetric measurements, hydrogen evolution, or other methods is the anomalous chemical dissolution process, occurring simultaneously with the normal electrochemical corrosion attack. Experimental evidence for this was shown by Kolotyrkin and coworkers through a large series of studies reporting proofs of direct chemical reaction of $\mathrm{H}_{2} \mathrm{O}$ molecules with the metallic surface without interference of electron transfer reactions [14-16]. Further details about the anomalous chemical dissolution of metals are available in a review article by Florianovich [17] and recent studies from Drazic and Propic [18, 19]. Since chemical reaction cannot be followed by electrochemical means, the electrochemical methods might give much smaller corrosion rates than those determined by weight loss measurements, volume of hydrogen gas, or amount of corrosion products in solution as a function of the immersion time.

Finally, the use of the Stern-Geary [20] equation (3) for the electrochemical estimation of the corrosion current density $\left(\mathrm{I}_{\text {corr }}\right)$,

$$
\mathrm{I}_{\text {corr }}=\frac{\mathrm{B}}{\mathrm{R}_{\mathrm{t}}}
$$

where $R_{t}$ is the charge transfer resistance, presents two main challenges: the precise knowledge of the B constant (4) and the value of the electrochemical equivalent of magnesium in order to calculate the mass loss from $\mathrm{I}_{\text {corr }}$ :

$$
\mathrm{B}=\frac{\mathrm{b}_{\mathrm{a}} \cdot \mathrm{b}_{\mathrm{c}}}{2.3\left(\mathrm{~b}_{\mathrm{a}}+\mathrm{b}_{\mathrm{c}}\right)} \text {. }
$$

The exact values of the anodic and cathodic Tafel slopes $\left(b_{a}\right.$ and $b_{c}$ ), which are required for the calculation of B (4), are often unknown. For magnesium and its alloys immersed in a sodium borate solution, Hoey and Cohen [4] determined $a b_{a}$ value of $\sim 60 \mathrm{mV}$ from polarization data and assumed $\mathrm{a}_{\mathrm{c}}$ value of $\sim 120 \mathrm{mV}$ (i.e., $\mathrm{B} \sim 0.018 \mathrm{~V}$ ), though these values were questioned by Song et al. [21]. Pebere et al. [22], using a sodium sulphate solution, found values of $b_{a} \sim$ $208 \mathrm{mV}$ and $b_{\mathrm{c}} \sim 280 \mathrm{mV}(\mathrm{B}=0.052 \mathrm{~V})$. Mathieu et al. [23] deduced a value of $\sim 0.075 \mathrm{~V}$ from the correlation between $\mathrm{R}_{\mathrm{t}}$ and mass loss tests in a solution containing sulphate, bicarbonate, and chloride of sodium. In addition to these significant variations in the value of $B$, the uncertainty of the electrochemical estimates is also affected by the mentioned possible participation of divalent and monovalent ions in the magnesium dissolution reactions $[8,24,25]$.

Taking into consideration the aforementioned issues, in the present study, the electrochemical estimation of the corrosion rate was investigated in the case of magnesium/aluminium alloys immersed in $3.5 \mathrm{wt} . \% \mathrm{NaCl}$ aqueous solution. In particular, the effect of the alloy composition in the magnitude of the discrepancies between electrochemical and gravimetric corrosion values was evaluated.

\section{Experimental}

2.1. Test Materials. The chemical compositions of the tested magnesium alloys, AZ31, AZ80, and AZ91D, are listed in Table 1. Unalloyed $\mathrm{Mg}$ was used as the reference material. The Mg and AZ31 materials were fabricated in wrought condition and supplied in plates of $3 \mathrm{~mm}$ thickness. The AZ80 and AZ91D alloys were manufactured by casting process and used in the experiments as billets of $300 \times$ 10 and $250 \times 10 \mathrm{~mm}$, respectively. All the materials were supplied by Magnesium Elektron Ltd. Details about their metallographic characterization were reported previously [26].

2.2. Electrochemical Measurements. The electrochemical impedance measurements were made with an AUTOLABPGSTAT 30 potentiostat with Frequency response analyzer (FRA) software. The frequency ranged from $100 \mathrm{kHz}$ to $1 \mathrm{mHz}$ with 5 points/decade and the amplitude of the sinusoidal potential signal was $10 \mathrm{mV}$ with respect to the open circuit potential (OCP), which remained stable before the electrochemical measurements. A three-electrode set-up was employed: the working electrode (or studied material), with an immersed area of $2 \mathrm{~cm}^{2}$, and $\mathrm{Ag} / \mathrm{AgCl}$ (SSE) and graphite as the reference and counter electrodes, respectively. 
TABLE 2: Comparison between direct mass losses and the corresponding electrochemical estimates.

\begin{tabular}{|c|c|c|c|c|}
\hline Material & Time & $\begin{array}{l}\text { Directly measured } \\
\text { mass losses } \\
\left(\mathrm{mg} / \mathrm{cm}^{2}\right)\end{array}$ & $\begin{array}{c}\text { Electrochemical } \\
\text { estimates } \\
\left(\mathrm{mg} / \mathrm{cm}^{2}\right)\end{array}$ & Ratio \\
\hline \multirow{3}{*}{$\mathrm{Mg}$} & $1 \mathrm{~h}$ & 13.04 & 1.37 & 9.52 \\
\hline & $3 \mathrm{~h}$ & 39.09 & 5.14 & 7.61 \\
\hline & $6 \mathrm{~h}$ & 78.18 & 16.00 & 4.89 \\
\hline \multirow{3}{*}{ AZ31 } & $1 \mathrm{~d}$ & 5.52 & 3.36 & 1.64 \\
\hline & $7 \mathrm{~d}$ & 38.6 & 23.91 & 1.61 \\
\hline & $10 \mathrm{~d}$ & 55.4 & 34.84 & 1.59 \\
\hline \multirow{3}{*}{ AZ80 } & $1 \mathrm{~d}$ & 0.074 & 0.19 & 0.39 \\
\hline & $7 \mathrm{~d}$ & 0.52 & 1.30 & 0.40 \\
\hline & $10 \mathrm{~d}$ & 0.74 & 1.85 & 0.40 \\
\hline \multirow{3}{*}{ AZ91D } & $1 \mathrm{~d}$ & 0.088 & 1.11 & 0.079 \\
\hline & $7 \mathrm{~d}$ & 0.62 & 7.64 & 0.081 \\
\hline & $10 \mathrm{~d}$ & 0.89 & 10.82 & 0.082 \\
\hline
\end{tabular}

The test medium was $3.5 \mathrm{wt} . \% \mathrm{NaCl}$ naturally aerated solution at room temperature $\left(22^{\circ} \mathrm{C}\right)$ and the immersion time was up to 28 days. Duplicate measurements were conducted to ensure reproducible test results.

2.3. Gravimetric Measurements. The gravimetric measurements were performed using specimens of working area $\sim 16 \mathrm{~cm}^{2}$ immersed in $3.5 \mathrm{wt} . \% \mathrm{NaCl}$ solution ( $\mathrm{pH} 5.6$ ) at room temperature. The $\mathrm{pH}$ freely evolved during the test. Prior to the tests, the specimens were measured and weighed. Once the test was finished for each immersion time, the samples were extracted, rinsed with isopropyl alcohol, dried in hot air, and then weighed again in order to calculate the mass loss per unit surface area. The specimens were weighed periodically during 10 days. After this time the morphology and composition of the corrosion products layer adhered to the surface were probed [26]. The gravimetric measurements were reproduced twice.

\section{Results and Discussion}

3.1. Electrochemical Results. Figure 1 discloses the Bode diagrams of the tested materials after immersion in $3.5 \mathrm{wt} . \%$ $\mathrm{NaCl}$ for 1 to 28 days. In general, the diagrams comprised two impedance arrests at low and high frequencies and a diagonal straight line of a slope of -1 at intermediate frequencies. The marked irregularities shown in Figure 1(a) for the magnesium metal indicated irreversible changes occurring in the exposed surface during impedance measurements due to its exceptionally high corrosion rate.

The impedance diagrams were interpreted in regard to the combined effect of the charge transfer resistance, $R_{t}$, of the corrosion process and a capacitance in parallel. The $\mathrm{R}_{\mathrm{t}}$ values were given by the difference between the impedance arrests at high and low frequencies, the latter corresponding to the ohmic resistance of the solution, mainly that of the electrolyte in the connected pores through the layer of corrosion products on the metal surface. With increasing immersion time higher values of $\mathrm{Z}$ for the horizontal portion at high frequencies were observed, which are possibly associated with the thickening of the corrosion layer. Further, from the calculated $R_{t}$ values and the Stern-Geary equation, the average corrosion values of the various tested materials were estimated (Table 2, column 4). Values agreed within $\sim 20 \%$ for duplicated specimens. The values for magnesium metal, deduced in this case from very irregular diagrams, should be viewed only as an approximated trend. Depending on the material, $R_{t}$ values and the calculated mass losses can differ by up to two orders of magnitude. A previous work by Pardo et al. [26] already revealed the influence of chemical composition and microstructure on the corrosion behaviour of these magnesium-based materials. The different ratios between directly measured mass losses and electrochemical estimates (Table 2, column 5) suggested that the electrochemical methods underestimated the corrosion rate of $\mathrm{Mg}$ and the AZ31 alloy and gave more reliable values in the case of the AZ80 and AZ91D alloys (see Section 3).

3.2. Gravimetric Results. The gravimetric mass losses followed approximately linear kinetics with the immersion time up to 10 days. The experimental data were fitted to a linear equation of the type " $y=k \cdot t$ ", where " $y$ " represents the mass loss in $\mathrm{mg} \mathrm{cm}^{-2}$, " $t$ " is the time in hours, and " $k$ " is the kinetic constant of the corrosion process. This constant disclosed values of $13.03 \mathrm{mg} \mathrm{cm}^{-2} \mathrm{~h}^{-1}$ for $\mathrm{Mg}$ in the interval $0 \leq t \leq 16 \mathrm{~h}$ and of $2.31 \times 10^{-1}, 3.10 \times 10^{-3}$, and $3.70 \times 10^{-3} \mathrm{mg} \mathrm{cm}^{-2} \mathrm{~h}^{-1}$ for the AZ31, AZ80, and AZ91D magnesium alloys in the interval $0 \leq t \leq 240 \mathrm{~h}$, respectively [26]. In Table 2 (column 3) several directly measured mass losses are given. Similarly to the electrochemical results, significant mass loss differences were revealed between the four tested materials, indicating the strong influence of the aluminium concentration and alloy microstructure on the corrosion susceptibility of the tested materials. The obtained gravimetric values can significantly differ from those of electrochemical measurements; that is, see electrochemical 


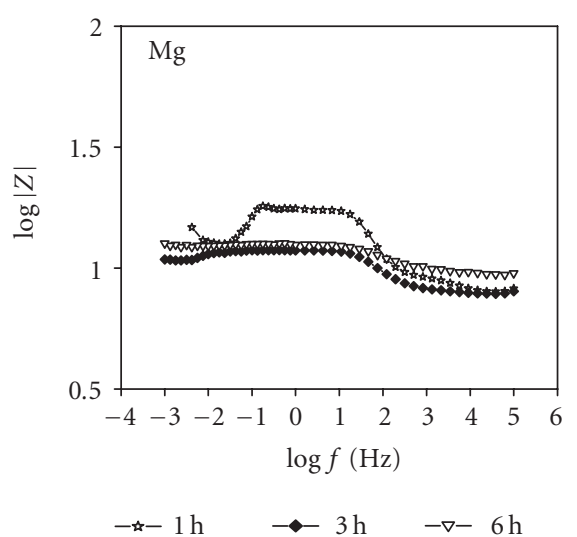

(a)

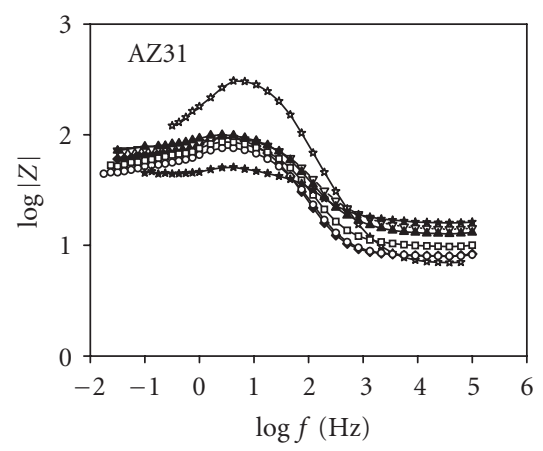

(c)

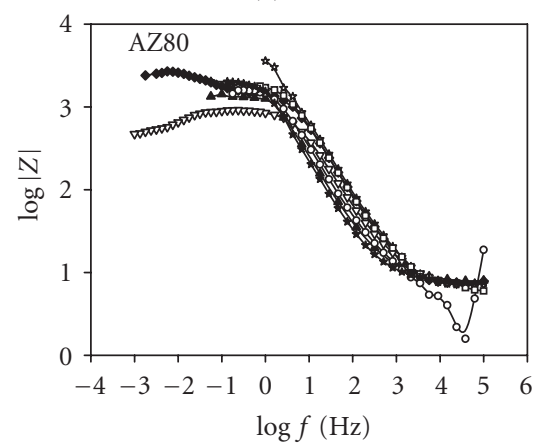

(e)
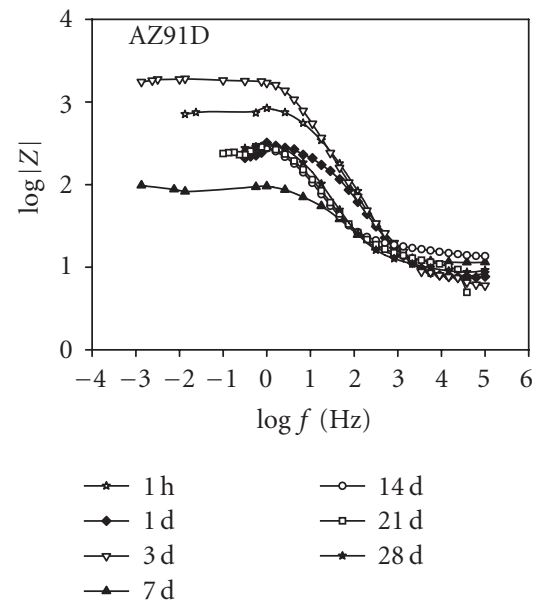

(g)

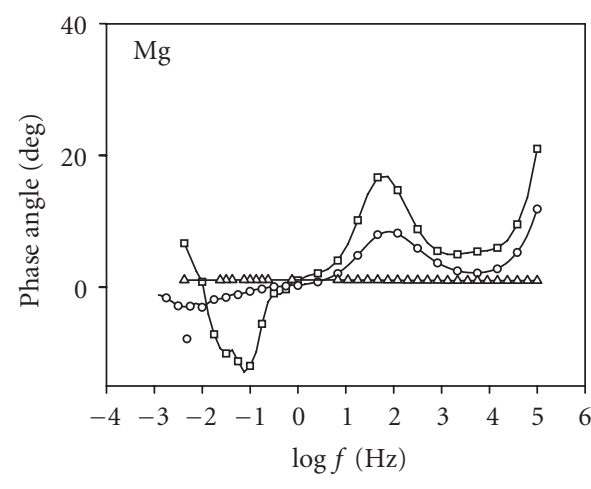

$\neg-1 \mathrm{~h} \quad \multimap-0 \mathrm{~h} \quad-\triangle 6 \mathrm{~h}$

(b)

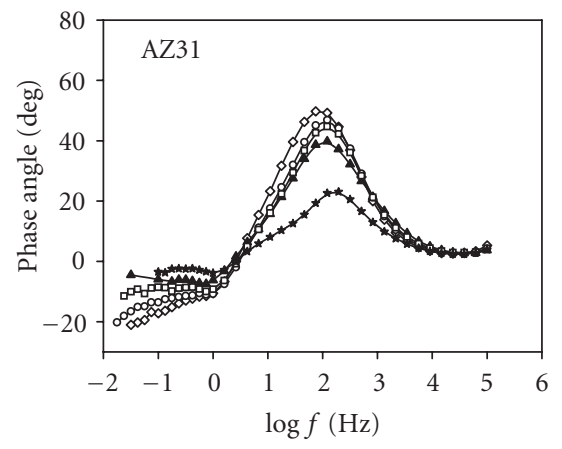

(d)

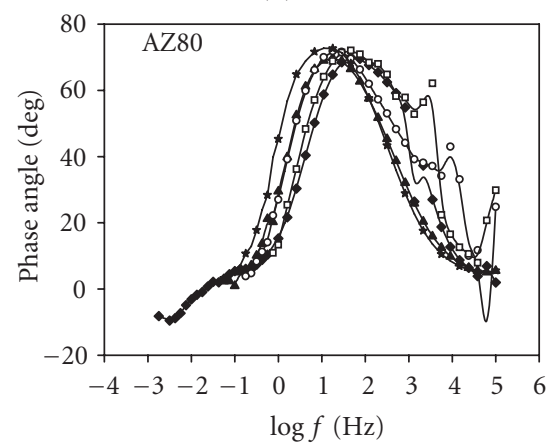

(f)

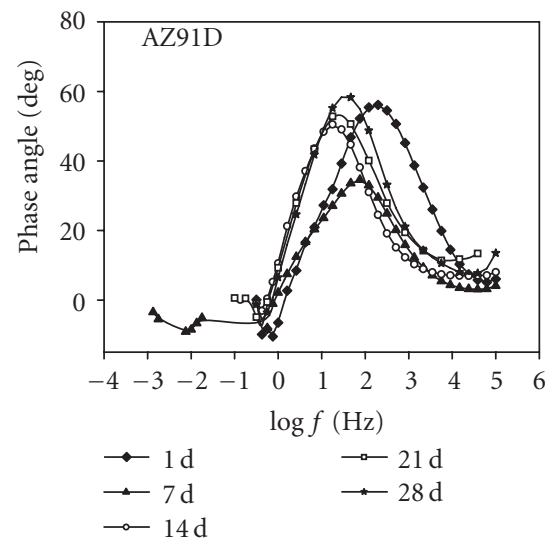

(h)

Figure 1: Impedance Bode diagrams as a function of immersion time in $3.5 \mathrm{wt} . \% \mathrm{NaCl}$ solution for tested specimens of (a) Mg metal and AZ31 alloy and (b) AZ80 and AZ91D alloys. 
TABLE 3: Representative thicknesses values of the corrosion layers adhered to the metal surfaces.

\begin{tabular}{lcc}
\hline Material & $\begin{array}{c}\text { Immersion } \\
\text { time }\end{array}$ & $\begin{array}{c}\text { Layer thickness } \\
(\mu \mathrm{m})\end{array}$ \\
\hline $\mathrm{Mg}$ & $5 \mathrm{~h}$ & 480 \\
$\mathrm{AZ31}$ & $28 \mathrm{~d}$ & 300 \\
$\mathrm{AZ80}$ & $28 \mathrm{~d}$ & 35 \\
AZ91D & $28 \mathrm{~d}$ & 150 \\
\hline
\end{tabular}

data in Table 2, column 4, corresponding to Approach I (see later).

The nucleation and growth of corrosion products adhered to the surface (Table 3), mainly consisting of $\mathrm{Mg}(\mathrm{OH})_{2}$ [26], resulted in lower gravimetric mass losses than the real amount of metal corroded during immersion in the $3.5 \mathrm{wt} . \% \mathrm{NaCl}$ solution. In Table 4 , column 3, the directly measured mass loss values were corrected taking into consideration the average thickness of the corrosion layer (from examination of the cross-sections by SEM) and the density of $\mathrm{Mg}(\mathrm{OH})_{2}$. The corrected gravimetric values were $\sim 9$ times higher than the electrochemical ones in the case of pure $\mathrm{Mg}$, nearly twice bigger for the AZ31 alloy, and similar for the AZ80 and AZ91D alloys with higher aluminium concentration (columns 4-6 in Table 4). Thus, despite of this correction, the discrepancy between gravimetric and electrochemical values remained relatively high for some of the studied materials.

3.3. Application of the Stern-Geary Equation. In order to compare the electrochemical and gravimetric results, the significance of possible errors introduced during the measurements needed to be taken into consideration. The gravimetric mass losses were obtained by using a laboratory analytical balance (Sartorius BP 211D) with a precision of $0.1 \mathrm{mg}$, and the amount of corrosion products was relatively insignificant compared with the amount of metal loss. Therefore, a more important role has to be credited to the precision of the electrochemical measurements.

For the B constant, an approximated value of $50 \mathrm{mV}$ was derived from the Tafel slopes of the polarization curves obtained for the investigated magnesium-based materials immersed in 3.5 wt.\% NaCl [26] (Figure 2). These curves disclosed well-defined cathodic branches, but the anodic branches were not clear, indicating that the accuracy of the above $B$ value is questionable.

An alternative empiric estimation of B from the correlation between electrochemical and gravimetric measurements $[27,28]$ is only appropriate if concurrent with the electrochemical corrosion there were no other corrosion processes of comparable rate, for example, metal disintegration. For instance, this estimation would not be possible in the case of commercially pure $\mathrm{Mg}$ due to the highly uneven corrosion attack suggesting undermining of metallic particles during the corrosion process (Figure 3 ). Therefore, for the empiric estimation of $\mathrm{B}$, the electrochemical and gravimetric results of the AZ91D were used as they revealed small differences

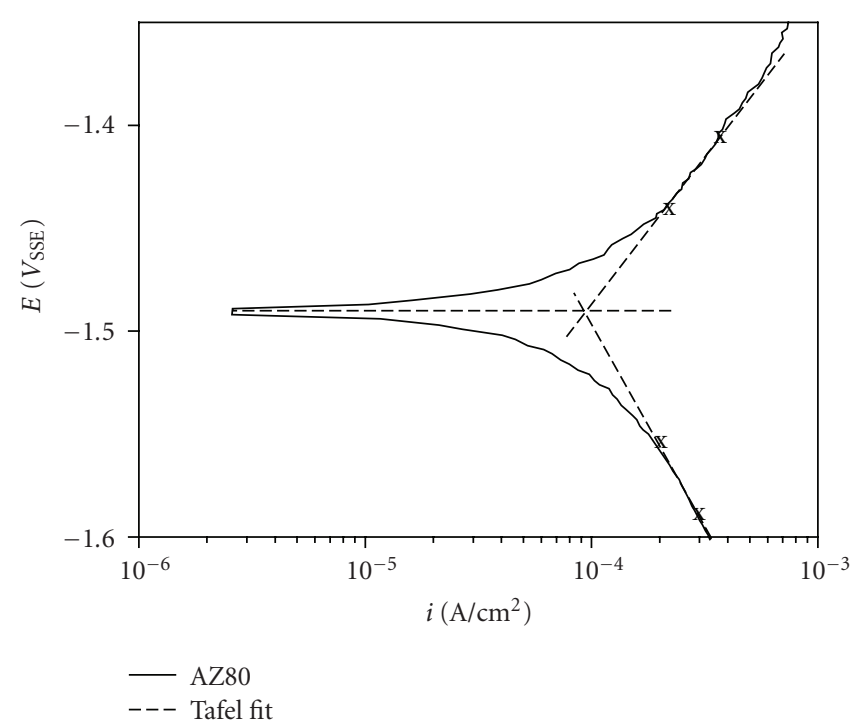

Figure 2: Example of Tafel fitting. Polarization curve of the AZ80 alloy after immersion in $3.5 \mathrm{wt} . \% \mathrm{NaCl}$ for 7 days.

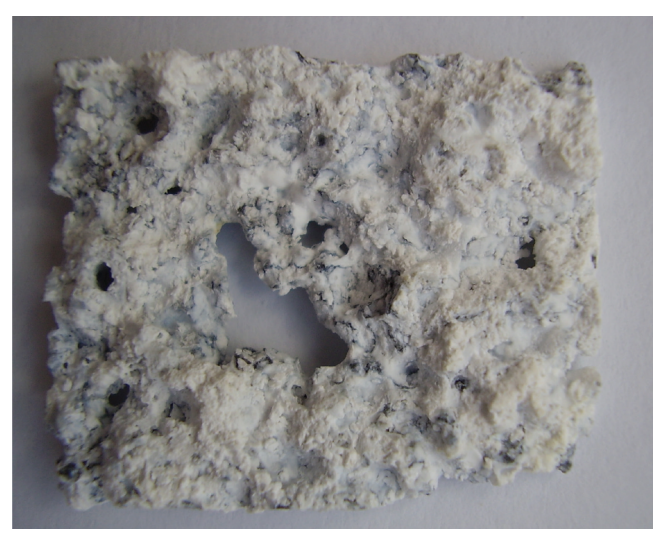

FIGURE 3: Uneven corrosion attack of pure Mg specimen after immersion in $3.5 \mathrm{wt} . \% \mathrm{NaCl}$ solution.

among them. Assuming that the gravimetric mass losses of the AZ91D alloy were exclusively due to the electrochemical dissolution of magnesium in the form of $\mathrm{Mg}^{2+}$, the calculated empirical B constant was $64 \mathrm{mV}$ or, in general, a B/n value of $32 \mathrm{mV}$, where " $n$ " is the valence.

To convert $\mathrm{I}_{\text {corr }}$ into mass loss values through Faraday's law, a fist approximation was to assume the formal valence of two for the magnesium ions. However, many references in literature have mentioned the presence of monovalent magnesium ions as an intermediate species in the anodic dissolution of this metal, and various authors $[8,24,25]$ determined the average valence of dissolved magnesium in a number of aqueous electrolytes, with the resultant values in the range of 1.33-1.66.

Therefore, even though a precise value of the B constant is difficult to obtain, the following estimations, based on the three different approaches, were expected to be reasonably close to the real corrosion values: 
TABLE 4: Average corrosion rate for samples immersed in $3.5 \mathrm{wt} . \% \mathrm{NaCl}$ solution. Test duration was 6 hours for Mg metal and 10 days for AZ31, AZ80 and AZ91D alloys.

\begin{tabular}{lccccc}
\hline Material & $\begin{array}{l}\text { Direct gravimetric } \\
\text { measurements }\left(\mathrm{mg} \mathrm{cm}^{-2} \mathrm{~d}^{-1}\right)\end{array}$ & \multicolumn{3}{c}{$\begin{array}{l}\text { Corrected gravimetric } \\
\text { measurements }\left(\mathrm{mg} \mathrm{cm}^{-2} \mathrm{~d}^{-1}\right)\end{array}$} & \multicolumn{3}{c}{ Electrochemical estimation $\left(\mathrm{mg} \mathrm{cm}^{-2} \mathrm{~d}^{-1}\right)$} \\
Approach I & 772 & 64 & 85 & Approach II & Approach III \\
\hline Mg & 310 & 8.1 & 3.5 & 4.7 & 82 \\
AZ31 & 5.5 & 0.37 & 0.19 & 0.25 & 0.24 \\
AZ80 & 0.074 & 1.4 & 0.10 & 1.47 \\
AZ91D & 0.089 & & & 1.40 \\
\hline
\end{tabular}

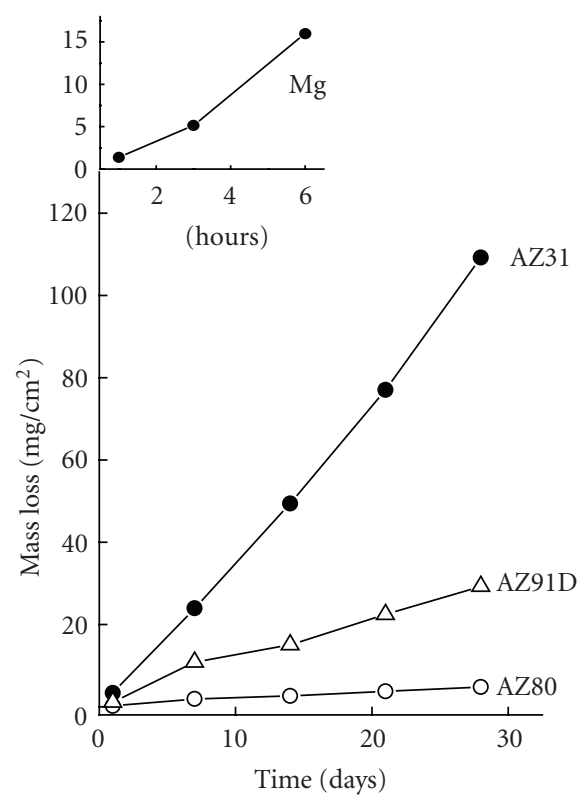

Figure 4: Electrochemically estimated corrosion losses of the materials immersed in $3.5 \mathrm{wt} . \% \mathrm{NaCl}$ solution.

Approach I. B $=50 \mathrm{mV}$ (determined from Tafel slopes), and the formal valence of 2 for the electrochemical equivalent of magnesium,

Approach II. $\mathrm{B}=50 \mathrm{mV}$ and an average value of $n=1.5$ for the valence of magnesium (monovalent and divalent ions in the same proportion),

Approach III. $\mathrm{B} / n=32 \mathrm{mV}$ (empirical estimation). The electrochemical mass loss data plotted in Figure 4 were obtained by numerical integration of electrochemical corrosion rate over the immersion time assuming Approach I. The other two approaches yielded graphs (not presented here) almost identical to those in Figure 4. Most of the points lie close to straight lines, indicating that corrosion kinetics for the tested materials was practically independent of the growth of corrosion products on the exposed surface. The metallographic characterization of the latter was reported previously [26]. The linear dependence with time suggested that the bulk of the film was porous and permeable to reacting species; hence, the film did not control de corrosion process. In the case of pure $\mathrm{Mg}$, the corrosion attack even accelerated with increasing immersion times (Figure 4), possibly due to an increase of the active exposed area of the base metal at the pores within the corrosion film. It is remarkable that this behaviour contradicted the most common one, where the rate of the attack tends to diminish as a result of the accumulation of reaction products.

Regarding the electrochemical estimates of corrosion in Table 4 , it should be noted that the above assumption of a combined effect of univalent and divalent magnesium ions in the electrochemical dissolution (Approach II) was supported by almost coinciding values in columns 5 and 6 of Table 4 .

It is evident that the electrochemical estimates of the corrosion damage of magnesium-based materials involve some degree of uncertainty. However, the electrochemical estimates are especially useful when one takes a relative, rather than absolute, point of view in judging the corrosion susceptibility of the materials. For instance, in Figure 5, the gravimetric losses (line 1) and the electrochemical estimates (line 2) of corrosion follow parallel lines.

3.4. Magnitude of the Discrepancy between Gravimetric and Electrochemical Tests. Stern and Weisert [29] have pointed out that even without a precise knowledge of the Tafel slopes, corrosion rates can be estimated within a 50\% error. Such degree of imprecision for the values of the B constant (3) can be also considered in the present study. Another source of error is the magnesium valence chosen for the conversion of $\mathrm{I}_{\text {corr }}$ into mass loss through Faraday's law in the interval between 2, for the normal case, and 1 in the case of monovalent $\mathrm{Mg}$ ions. However, these errors were not sufficient to explain the significant lower corrosion rate from the electrochemical measurements in the case of pure $\mathrm{Mg}$ compared with the gravimetric data (Table 4). This suggested that the corrosion system produced an "abnormal" response during the impedance spectroscopy measurements of this material. A similar conclusion can be drawn for the AZ31 alloy, although in this case the differences between electrochemical and gravimetric measurements were lower. On the other hand, the AZ80 and AZ91D alloys showed relatively good agreement between the electrochemical and gravimetric values (Table 4 ).

An anomalous response will take place when a fraction of the metallic surface exposed to the corrosive medium does not contribute to the electrochemical response of the corrosion system, due to, for example, disintegration of part of the metal surface into fine metallic particles, or to a simultaneous and independent process of chemical 


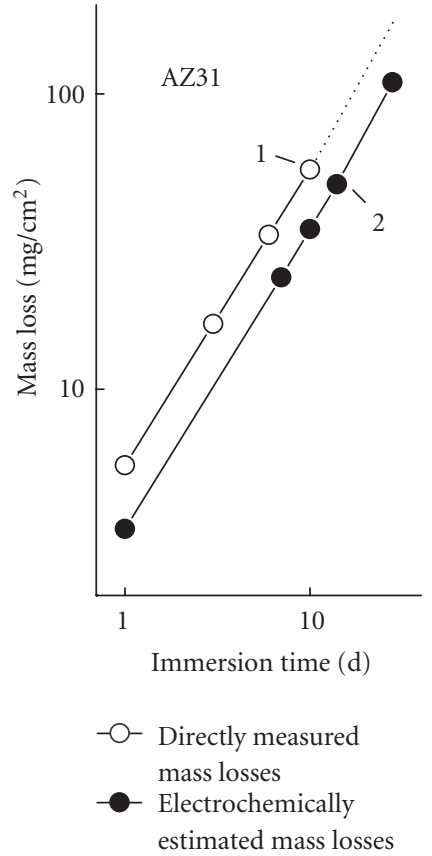

(a)

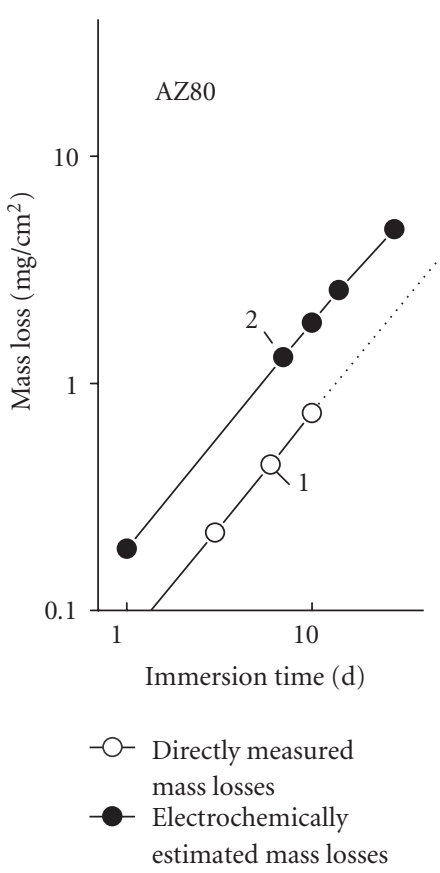

(b)

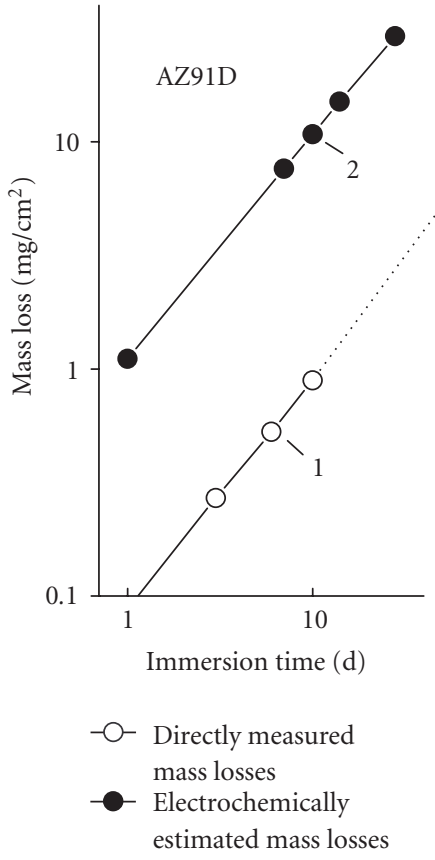

(c)

FIgURe 5: Variation of mass losses with time

TABLE 5: Relative importance of the anomalous response effect. AR percentages estimated on the assumption of Approaches I, II, and III.

\begin{tabular}{lccc}
\hline Material & Approach I & Approach II & Approach III \\
\hline Mg & 91.7 & 89.0 & 89.4 \\
AZ31 & 56.8 & 42.0 & 44.4 \\
AZ80 & 48.6 & 32.4 & 35.1 \\
AZ91D & 21.4 & $\sim 0$ & 0 \\
\hline
\end{tabular}

dissolution that cannot either be followed by electrochemical means. The intense hydrogen evolution during corrosion of magnesium may also be associated with the anomalous response as a result of temporally isolated regions of the surface due to the formation of hydrogen bubbles. Thus, further studies are needed in order to fully understand the origin of such discrepancies.

The relative importance of the anomalous response (AR) effect can be expressed in a quantitative way by using

$$
\mathrm{AR}=\frac{100(\mathrm{G}-\mathrm{E})}{\mathrm{G}}
$$

in which, G is the gravimetric value and $\mathrm{E}$ is the electrochemical estimation of corrosion. The AR values for the investigated materials are presented in Table 5. Even though the results according to Approaches I, II, and III were not entirely in agreement, some general conclusions were revealed. Firstly, the pure $\mathrm{Mg}$ specimen revealed a high AR value ( $\sim 90 \%)$, indicating that the electrochemical measurements gave a distorted vision of the damage suffered by this material. Secondly, the AZ31 and AZ80 alloys revealed a relatively important AR effect and, finally, this effect was almost insignificant in the case of the AZ91D magnesium alloy. However this alloy revealed a higher corrosion rate than that of the AZ80 alloy, indicating that there is no necessarily a direct relation between the magnitude of the AR value and the corrosion rate (Tables 4 and 5).

\section{Conclusions}

(1) The gravimetric mass losses and electrochemical estimates of the corrosion rate were compared for specimens of commercially pure magnesium and AZ31, AZ80, and AZ91D magnesium/aluminium alloys. The findings revealed discrepancies between the corrosion values determined from both methods, suggesting an anomalous response of the specimens to the applied electrochemical signal during electrochemical measurements. This anomalous electrochemical behaviour of the metallic surface was more significant for the commercially pure $\mathrm{Mg}$ and AZ31 materials and less pronounced for the AZ80 and AZ91D alloys with higher amounts of aluminium (89 wt.\%).

(2) The application of the Stern-Geary equation with B values in the range of $0.050-0.064 \mathrm{~V}$ gave corrosion rate values similar to those obtained by gravimetric measurements in the case of the AZ80 and AZ91D magnesium alloys. However, the electrochemical estimates for commercially pure $\mathrm{Mg}$ and the AZ31 alloy were not reliable and tended to underestimate the corrosion losses. 


\section{Acknowledgments}

The authors wish to thank both the MCYT (Project MAT2006-13179-C02-01-02) and the Community of Madrid through the program ESTRUMAT_CM (reference MAT/77) for the financial support to carry out this investigation.

\section{References}

[1] G. L. Makar and J. Kruger, "Corrosion studies of rapidly solidified magnesium alloys," Journal of the Electrochemical Society, vol. 137, no. 2, pp. 414-421, 1990.

[2] M. E. Straumanis and B. K. Bathia, "Disintegration of magnesium while dissolving anodically in neutral and acidic solutions," Journal of the Electrochemical Society, vol. 110, no. 5, pp. 357-360, 1963.

[3] W. J. James, M. E. Straumanis, B. K. Bathia, and J. W. Jhonson, "The difference effect on magnesium dissolving in acids," Journal of the Electrochemical Society, vol. 110, no. 11, pp. 1117-1120, 1963.

[4] G. R. Hoey and M. Cohen, "Corrosion of anodically and cathodically polarized magnesium in aqueous media," Journal of the Electrochemical Society, vol. 105, no. 5, pp. 245-250, 1958.

[5] G. L. Song and A. Atrens, "Corrosion mechanisms of magnesium alloys," Advanced Engineering Materials, vol. 1, no. 1, pp. 11-33, 1999.

[6] L. L. Shreir, Corrosion, George Newness, London, UK, 1965.

[7] G. G. Perrault, "The potential-pH diagram of the magnesiumwater system," Journal of Electroanalytical Chemistry, vol. 51, no. 1, pp. 107-119, 1974.

[8] R. L. Petty, A. W. Davidson, and J. Kleinberg, "The anodic oxidation of magnesium metal: evidence for the existence of unipositive magnesium," Journal of the American Chemical Society, vol. 76, no. 2, pp. 363-366, 1954.

[9] E. J. Casey, E. Bergeroy, and G. D. Nagt, "On the mechanism of dissolution of magnesium in aqueous magnesium chloride solutions-part II," Canadian Journal of Chemistry, vol. 40, no. 3, pp. 463-479, 1962.

[10] G. Song, A. Atrens, D. Stjohn, J. Nairn, and Y. Li, "The electrochemical corrosion of pure magnesium in $1 \mathrm{~N} \mathrm{NaCl}$," Corrosion Science, vol. 39, no. 5, pp. 855-875, 1997.

[11] G. Song, A. Atrens, and M. Dargusch, "Influence of microstructure on the corrosion of diecast AZ91D," Corrosion Science, vol. 41, no. 2, pp. 249-273, 1998.

[12] G. Song, A. Atrens, D. St John, X. Wu, and J. Nairn, "The anodic dissolution of magnesium in chloride and sulphate solutions," Corrosion Science, vol. 39, no. 10-11, pp. 19812004, 1997.

[13] G. Baril and N. Pébère, "Corrosion of pure magnesium in aerated and deaerated sodium sulphate solutions," Corrosion Science, vol. 43, no. 3, pp. 471-484, 2001.

[14] Ya. M. Kolotyrkin and G. M. Florianovich, Zashchita Metallov, vol. 1, p. 7, 1965.

[15] Ya. M. Kolotyrkin and T. R. Agladze, Zashchita Metallov, vol. 4, p. 413, 1967.

[16] Ya. M. Kolotyrkin and G. M. Florianovich, Elektrokhimiya, vol. 9, p. 413, 1973.

[17] G. M. Florianovich, "Electroless dissolution of metals: substantiation and alternative notions," Russian Journal of Electrochemistry, vol. 36, no. 10, pp. 1037-1042, 2000.
[18] D. M. Dražić and J. P. Popic, "Anomalous dissolution of metals and chemical corrosion," Journal of the Serbian Chemical Society, vol. 70, no. 3, pp. 489-511, 2005.

[19] D. M. Dražić and J. P. Popić, "Electrochemistry of active chromium-part : anomalous corrosion and products of chromium dissolution in deaerated sulfuric acid," Corrosion, vol. 60, no. 3, pp. 297-303, 2004.

[20] M. Stern and A. L. Geary, "Electrochemical polarization," Journal of the Electrochemical Society, vol. 104, no. 1, pp. 5663, 1957.

[21] G. Song, A. Atrens, X. Wu, and B. Zhang, "Corrosion behaviour of AZ21, AZ501 and AZ91 in sodium chloride," Corrosion Science, vol. 40, no. 10, pp. 1769-1791, 1998.

[22] N. Pebere, C. Riera, and F. Dabosi, "Investigation of magnesium corrosion in aerated sodium sulfate solution by electrochemical impedance spectroscopy," Electrochimica Acta, vol. 35, no. 2, pp. 555-561, 1990.

[23] S. Mathieu, C. Rapin, J. Hazan, and P. Steinmetz, "Corrosion behaviour of high pressure die-cast and semi-solid cast AZ91D alloys," Corrosion Science, vol. 44, no. 12, pp. 2737-2756, 2002.

[24] P. Brouillet, I. Epelboin, and M. Froment, Comptes-Rendus Hebdomadaires des Seances de l'Académie des Sciences, vol. 39, p. 1795, 1954.

[25] J. Przluski and E. Palka, "Untersuchung der Kinetik der anodischen oxydation des magnesiums in einer ammoniumchloridlösung," Electrochimica Acta, vol. 15, no. 5, pp. 853864, 1970.

[26] A. Pardo, M. C. Merino, A. E. Coy, R. Arrabal, F. Viejo, and E. Matykina, "Corrosion behaviour of magnesium/aluminium alloys in 3.5 wt.\% NaCl," Corrosion Science, vol. 50, no. 3, pp. 823-834, 2008.

[27] M. Prazák, "The polarization resistance method for corrosion testing," Materials and Corrosion, vol. 25, no. 2, pp. 104-112, 1974.

[28] P. J. Moreland and J. C. Rowlands, "Technique and instrumentation for polarisation resistance measurements," British Corrosion Journal, vol. 12, no. 2, pp. 72-79, 1977.

[29] M. Stern and E. D. Weisert, "Experimental observations on the relation between polarization resistance and corrosion rate," Proceedings of the American Society for Testing and Materials, vol. 59, pp. 1280-1291, 1959. 

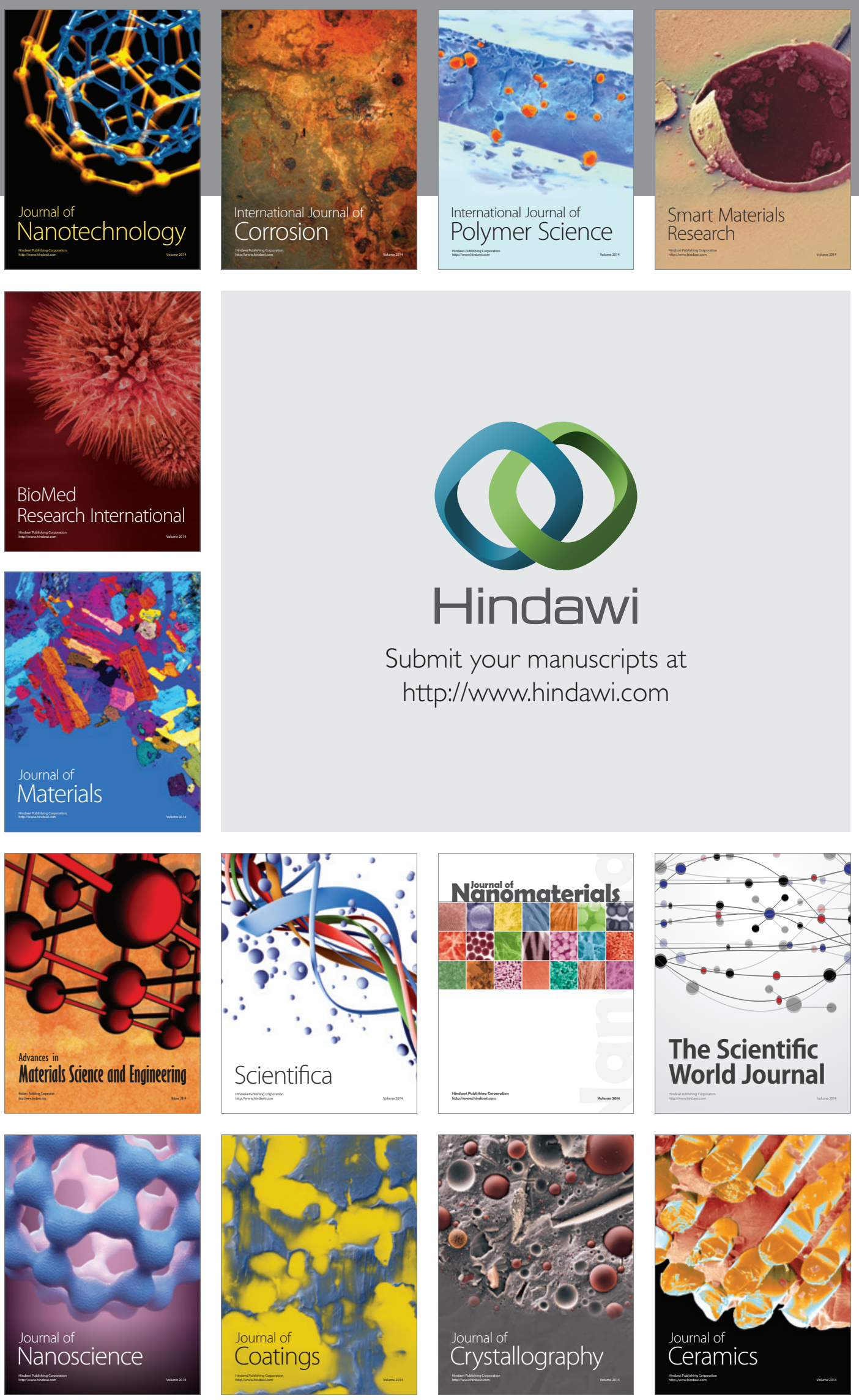

The Scientific World Journal

Submit your manuscripts at

http://www.hindawi.com

\section{World Journal}

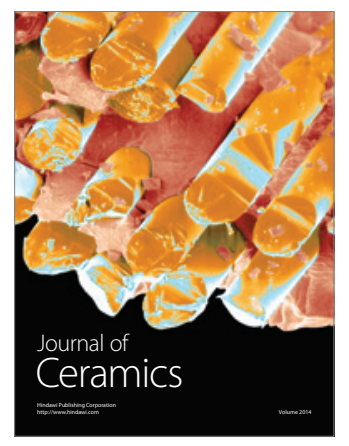

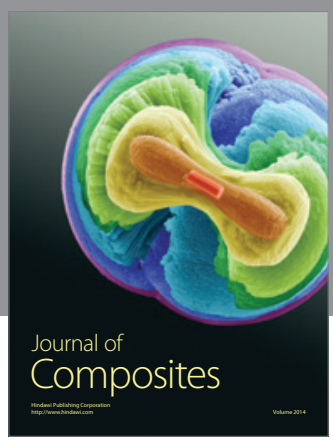
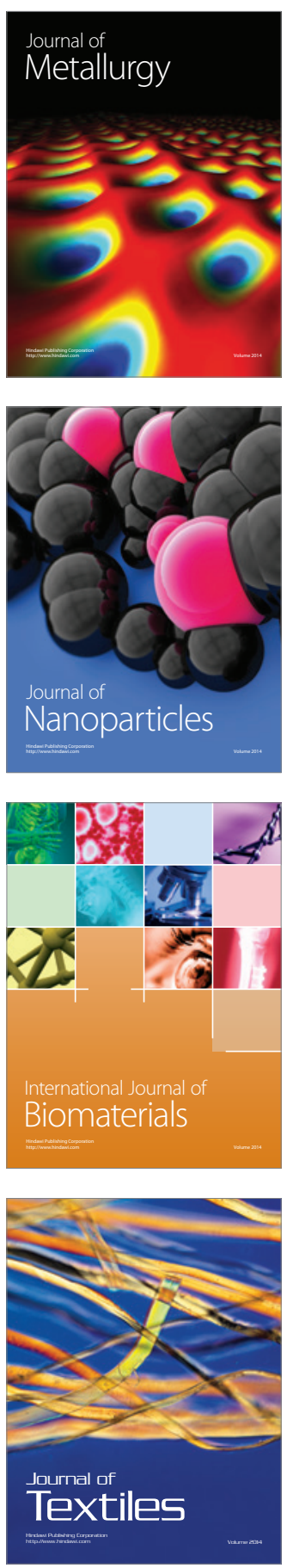